\title{
Qualitätssicherung beeinträchtigt die Sicherheit der Schwangerschaftsabbrüche in der Schweiz
}

\author{
André Seidenberg", \\ Anne-Marie Rey ${ }^{b}$ \\ a Präsident APAC \\ b Sekretariat APAC
}

- Schweizerische Gesellschaft für Gynäkologie und Geburtshilfe SGGG. Manual über das Beratungs-gespräch vor einem Schwangerschaftsabbruch. www.sggg.ch/ D/intern/expertenbriefe/ SSAB-manual-d.pdf.

1 WHO. Safe Abortion - Technical and Policy Guidance for Health Systems. WHO: Geneva; 2003. www.who.int/reproductive-health/ publications/safe abortion/safe abortion.pdf.

2 Royal College of Obstetricians and Gynaecologists RCOG. The Care of Women Requesting Induced Abortion. Evidence-based Clinical Guideline Number 7; Sept 2004. www.rcog.org.uk/resources/Public/ pdf/induced_abortionfull.pdf.

3 Agence Nationale d'Accréditation et d'Evaluation en Santé ANAES. Prise en charge de l'interruption de grossesse jusqu'à 14 semaines. Service de recommandation et références professionnelles, mars 2001; www.has-sante.fr/ portail/upload/docs/application/ pdf/IVGRAP.pdf.

\section{Korrespondenzen:}

Dr. med. André Seidenberg Facharzt Allgemeinmedizin FMH Weinbergstrasse 9 CH-8001 Zürich

andre.seidenberg@hin.ch

www.seidenberg.ch

Anne-Marie Rey

APAC-Suisse

Grabenstrasse 21

3052 Zollikofen

Tel. 0319115794

apac-suisse@bluewin.ch
Die Schweiz hat weltweit wohl die niedrigste Rate an Schwangerschaftsabbrüchen, vermutlich weil der Zugang zu Verhütungsmitteln und Schwangerschaftsabbrüchen allen Frauen gut offen steht. Ein besonders niederschwelliges Angebot besteht in der Durchführung von Schwangerschaftsabbrüchen in Lokalanästhesie in der Arztpraxis. Das Thema wurde am SGGG-Jahreskongress der gynécologie suisse in Interlaken im Juni dieses Jahres diskutiert.

Neue Vorschriften drohen jedoch diese Möglichkeit zu unterbinden. So wurden die Anforderungen für einen Praxisoperationssaal erheblich verschärft. Und der Kanton Waadt hat soeben Weisungen erlassen, die zwingend die Anwesenheit eines Anästhesisten verlangen, was nicht nur unnötig ist, sondern den Eingriff massiv verteuert. Der ambulante Schwangerschaftsabbruch in Lokalanästhesie in der Praxis wird damit praktisch verunmöglicht.

Oft stehen zweifelhafte Interessen hinter solchen neuen Anforderungen. Sie bringen keine bessere Qualität für einen so einfachen und risikoarmen Eingriff wie eine ambulante Saugcurettage in Lokalanästhesie. Vielmehr erschweren sie den kostengünstigen Zugang zu sicheren Schwangerschaftsabbrüchen, Frauen in Not werden wieder häufiger in gefährliche Situationen geraten.

In der Schweiz droht die Lokalanästhesie als sicherste Methode der Schmerzbekämpfung beim chirurgischen Schwangerschaftsabbruch bis zur 12. Woche in Vergessenheit zu geraten. Dies obwohl die WHO [1] wie auch die offiziellen britischen [2] und französischen Richtlinien [3] empfehlen, der Lokalanästhesie gegenüber Vollnarkosen für den Schwangerschaftsabbruch mit Saugcurettage den Vorzug zu geben. Es gibt keine Evidenz, die für die Vollnarkose beim Schwangerschaftsabbruch im ersten Trimenon spricht.

Die erwähnten Guidelines beziehen sich hauptsächlich auf eine sehr grosse, ältere CDCMortalitätsstudie [4, 5]. Deren Resultate wurden aber durch einige neuere kleinere Studien mit Morbiditätsendpunkten bestätigt [6-8].

Todesfälle durch Schwangerschaftsabbrüche sind in entwickelten Gesellschaften mit gutem

\section{L'assurance-qualité compromet}

\section{la sécurité des avortements}

La Suisse a le plus bas taux d'avortements au monde, probablement parce que l'accès aux moyens contraceptifs et à l'avortement est ouvert à toutes les femmes sans exception. Une offre particulièrement accessible représantent les avortements sous anesthésie locale au cabinet médical. Ce thème a été discuté au congrès annuel de la Société suisse de gynécologie et d'obstétrique tenu en juin dernier à Interlaken.

De nouvelles prescriptions menacent toutefois de mettre un terme à cette possibilité. Les exigences relatives à une salle d'opération au cabinet médical ont été considérablement renforcées. Le canton de Vaud a également promulgué des directives exigeant impérativement la présence d'un anesthésiste, ce qui est non seulement inutile mais renchérit énormément cette intervention. L'avortement ambulatoire sous anesthésie locale au cabinet médical est rendu pratiquement impossible.

On trouve souvent des intérêts douteux derrière ce genre d'exigences nouvelles. Elles n'amènent aucune amélioration de la qualité pour une intervention aussi simple et sans risque que le curetage ambulatoire par aspiration sous anesthésie locale. Au contraire, elles entravent l'accès bon marché à des avortements effectués en toute sécurité et les femmes en détresse tombent plus souvent dans des situations dangereuses.

Zugang zu legalen ärztlichen Behandlungen sehr selten: In den USA waren es insgesamt etwa 8,5 Todesfälle pro 1 Million Schwangerschaftsabbrüche. Mit Vollanästhesie starben gemäss der CDC-Studie fast viermal mehr Frauen durch 


\section{Abbildung 1}

Der Vergleich der in den verschiedenen Phasen der Behandlung empfundenen Schmerzen [11] zeigt das akzeptable Niveau der Analgesie durch lokale Infiltrationstechniken deutlich. Dilatationsschmerzen sind beispielsweise mit Menstruationsschmerzen vergleichbar.

Schmerz von 0-10

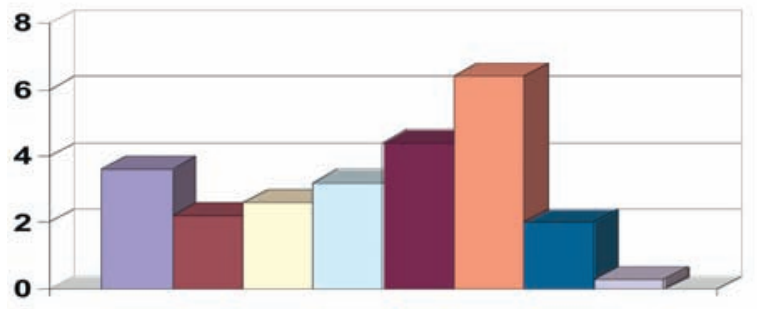

$\square$ Menstruationsschmerzen

Schmerz durch Infusion

Schmerz durch Speculum

Schmerz durch Infiltration

Schmerz durch Dilatation

Schmerz nach Operation

Schmerz nach 1 Stunde

Schmerz beim Ausgang
4 Peterson HB, Grimes DA, Cates W, Rubin GL. Comparative risk of death from induced abortion at $\checkmark 12$ weeks' gestation performed with local versus general anesthesia. Am J Obstet Gynecol. 1981;141:763-8.

5 Lawson HW et al. Abortion mortality, United States, 1972 through 1987. Am J Obstet Gynecol. 1994;171:1365-72.

6 Osborn JF, Arisi E, Spinelli A, Stazi MA. General anaesthesia, a risk factor for complication following induced abortion? Eur J Epidemiol. 1990;6:416-22.

7 Thonneau P, Fougeyrollas B, Ducot B, Boubilley D, Dif J, Lalande M, Soulat C. Complications of abortion performed under local anesthesia. Eur J Obstet Gynaecol Reprod Biol. 1998;81:59-63.

8 Pons JC, Vendittelli F, Lachcar P. L'interruption volontaire de grossesse et sa prévention. Paris: Masson; 2004.

9 Soulat C, Gelly M. Immediate complications of surgical abortion. J Gynecol Obstet Biol Reprod (Paris). 2006;35(2):157-62.

10 Hern WM. Abortion Practice. Apenglo grafis, 1130 Alpine Boulder, Colorado, ISBN 0-9625728-0-2. 368 p, 1990

11 Ambassa Mballa N. Bloc paracervical versus injection intracervicale dans la prise de charge de la douleur au cours de l'interruption volontaire de grossesse sous anesthésie locale. Dissertation Université Lille II, Faculté de Médecine Henri Warembourg, 2007.

12 Bachelot A et al. Conditions for choosing between drug-induced and surgical abortions. Contraception. 1992;45:547-59.
Schwangerschaftsabbrüche, als wenn sie in Lokalanästhesie durchgeführt wurden. Zweifellos ist die Vollnarkose in den vergangenen drei Jahrzehnten besser geworden; aber das Verhältnis von etwa vier $\mathrm{zu}$ eins zeigte sich sowohl als direkt mit der Anästhesie zusammenhängende Folge als auch als indirekte Folge der Anästhesietechnik. Auch wenn nur Todesfälle durch nicht direkt mit der Anästhesietechnik zusammenhängende Ursachen analysiert wurden, starben 3,5-mal mehr Frauen beim Schwangerschaftsabbruch mit Narkose als mit Lokalanästhesie. Diese Zusammenhänge zeigten sich auch, wenn rechnerische Störfaktoren wie gleichzeitige Sterilisation, vorbestehende Krankheiten oder das Gestationsalter berücksichtigt wurden: Vollnarkose blieb mindestens 2,5-mal riskanter.

In den jüngeren Studien [6-8] wurden die deutlichen technischen Vorteile der Lokalanästhesie anhand von Morbiditätsendpunkten sichtbar. In Vollnarkosen (ohne Halothane) treten mehr Uterusperforationen, Zervixverletzungen und schwere Blutungen auf $[6,9]$.

Wichtig scheint uns, dass alle direkt durch Lokalanästhesie verursachten Todesfälle der CDCStudie durch Überdosierung des Lokalanästhetikums erfolgten. Erfahrung und eine gute Operationstechnik [10] sind immer entscheidend. Die Technik der Lokalanästhesie für den Schwangerschaftsabbruch wurde kürzlich in einer Dissertationsschrift umfassend dargestellt [11]; eine kurze Anleitung kann bei den Autoren bestellt werden.

Für die Analgesie ist eine Lokalanästhesie im ersten Trimenon objektiv genügend. Auch unabhängig von den niedrigeren Kosten ziehen viele Frauen den Eingriff in Lokalanästhesie einer
In der APAC-Suisse (Association de professionnels de l'avortement et de la contraception) haben sich Fachleute zusammengeschlossen, die im Bereich Schwangerschaftsabbruch tätig sind. Sie sind bestrebt, die Qualität bei der Durchführung des Schwangerschaftsabbruchs zu fördern, das niederschwellige Angebot zur Durchführung des medikamentösen und chirurgischen Schwangerschaftsabbruchs unter bestmöglichen Bedingungen auszudehnen, insbesondere das Angebot des chirurgischen Eingriffs unter Lokalanästhesie in der Praxis und in der Klinik zu erweitern.

Mitglieder der APAC-Suisse bieten Tutorate und Hospitationen zur Durchführung von Abbrüchen in Lokalanästhesie in der Praxis an:

- Regina Widmer und Helene Huldi, Frauenpraxis Runa in Solothurn (regina.elizabeth@bluewin.ch)

- Vroni Knuchel, Gruppenpraxis Paradies in Binningen (gruppenpraxisparadies@bluewin.ch)

- Theres Blöchlinger (frauenambi@bluewin.ch)

- Bruno Maggi (magoer@bluewin.ch)

- André Seidenberg (andre.seidenberg@hin.ch)

Narkose vor, wenn sie die Möglichkeit der Wahl haben [12]. Die Arztpraxis bietet zudem in der Regel einen intimeren Rahmen für die Durchführung des Eingriffs als das Spital. 